\title{
Metabolomics and metabolite profiling
}

\author{
Rainer Schuhmacher • Rudolf Krska • \\ Wolfram Weckwerth • Royston Goodacre
}

Published online: 17 April 2013

(C) Springer-Verlag Berlin Heidelberg 2013

During the last 15 years, metabolomics has emerged as the latest of the so-called "omics" research fields. It deals with the analytical characterization of the metabolome, i.e. the low molecular weight metabolite complement of the biological system under investigation. A wide range of biological systems have benefited from metabolomics studies, from relatively simple microbes to plants and complex multiorganism systems such as mammals. The general goal of most metabolomics studies is to generate a snapshot of the metabolic state of a biological sample and to characterize the changes in the abundances of the measured metabolites arising from natural fluctuations or external, experimental biotic or abiotic perturbations.

The topical collection in this issue of Analytical and Bioanalytical Chemistry provides a glimpse of the current

Published in the topical collection Metabolomics and Metabolite Profiling with guest editors Rainer Schuhmacher, Rudolf Krska, Roy Goodacre, and Wolfram Weckwerth.

R. Schuhmacher $(\bowtie) \cdot R$. Krska

Department for Agrobiotechnology (IFA-Tulln),

Center for Analytical Chemistry, University of Natural Resources

and Life Sciences, Vienna (BOKU), Konrad-Lorenz-Strasse 20,

3430 Tulln, Austria

e-mail: rainer.schuhmacher@boku.ac.at

W. Weckwerth

Department Molecular Systems Biology, Faculty of Life Sciences,

University of Vienna, Althanstrasse 14,

1090 Vienna, Austria

R. Goodacre

School of Chemistry, Manchester Institute of Biotechnology,

University of Manchester, Manchester M1 7DN, UK activities in metabolomics and metabolite profiling. The articles presented elegantly illustrate that the general concept of metabolomics and metabolite profiling has matured significantly during the last decade and that this recent "-omics" toolbox is widely applied in many scientific disciplines, such as diagnostics of human diseases, biomarker discovery, nutrition, food safety, plant science and microbiology.

Most state-of-the-art metabolomics studies use liquid chromatography (LC) or gas chromatography (GC) coupled with mass spectrometry (MS) for metabolite profiling, whereas direct infusion MS, NMR spectroscopy and IR spectroscopy are applied for metabolite fingerprinting without prior separation of the sample constituents. Other techniques such as LC with UV detection can be used to complement these techniques, as successfully illustrated by the contribution on carotenoid profiling of this issue. Moreover, the huge chemical diversity of substances contained in biological samples affords multiple analytical platforms in order to cover a wide range of metabolites. When more than one analytical method is applied, the data, which are derived from the metabolomics platforms, have to be integrated. In the present collection this topic has been addressed in the area of food safety with an untargeted GC-MS-based study using two different methods for the profiling of both volatiles and polar small metabolites for the detection of meat spoilage.

Independent of the analytical technique employed, two complementary approaches are widely used in current metabolomics: targeted and untargeted analyses. In the targeted approach a set of predefined known substances is monitored, which usually allows absolute quantification and definite identification when limited to metabolites available 
as authentic reference standards. In contrast, untargeted profiling methods try to find analytical features of all detectable compounds and therefore show the potential of probing the entire metabolic space, including substances which are currently unknown (or at least unidentified) at the time of measurement. Thus, untargeted approaches are suitable for detecting changes in unexpected parts of the metabolome and frequently lead to new scientific hypotheses. Especially in untargeted approaches, effective automated data processing, accurate quantification and reliable annotation/identification of metabolites still pose major challenges in metabolomics. In this context, stable-isotopeassisted profiling approaches offer great potential to tackle these challenges in the future. These highly interesting topics in the area of metabolomics have been addressed by several articles in this issue.

Moreover, the development and application of appropriate quality control measures, including quality control data processing algorithms, method validation and estimation of measurement uncertainty, are more and more being recognized as being crucial prerequisites for obtaining comparable results over longer time periods, across different sample batches and biological experiments or between different laboratories.

Thanks to the contributors, to whom we wish to express our great gratitude, this collection of articles is a timely reflection of significant current research in the area of metabolomics, including metabolite profiling and metabolite fingerprinting. We are also very thankful to the reviewers for their thorough and on-time reviews of the papers and to the editorial team of Analytical and Bioanalytical Chemistry for their highly professional and great cooperation. We hope you enjoy this special issue on metabolomics and metabolite profiling.

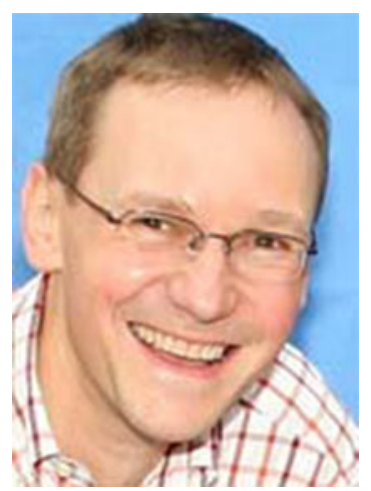

Rainer Schuhmacher

is Associate Professor in the Department for Agrobiotechnology (IFATulln) of the University of Natural Resources and Life Sciences, Vienna (BOKU). He currently heads the Metabolomics and Bioactive Compounds working group at BOKU, IFA-Tulln, and in the last few years he has shifted his research focus towards LC-MS- and GCMS-based metabolomics of microbes and plants. In 2012 he received the Fritz Feigl Award of the Austrian Society of Analytical Chemistry and he is the author or coauthor of more than 80 publications.

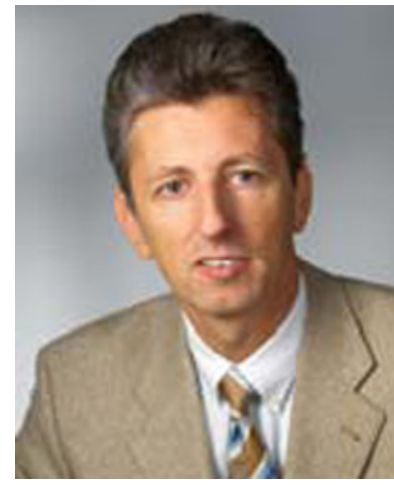

\section{Rudolf Krska}

is Professor of (Bio-)analytics and Organic Trace Analysis and is head of the Department for Agrobiotechnology (IFA-Tulln) at the University of Natural Resources and Life Sciences, Vienna (BOKU). In 2009-2010 he worked for 1 year as A/Chief of Health Canada's Food Research Division in Ottawa. He is an expert in food analysis by chromatographic and mass spectrometric techniques. In the last few years his research focus has been on metabolomics and metabolite pro-

filing to study plant-fungi interactions, an area which has been funded by the Austrian Science Fund within a Special Research Programme. He has received six scientific awards and is the author or coauthor of more than 180 publications.

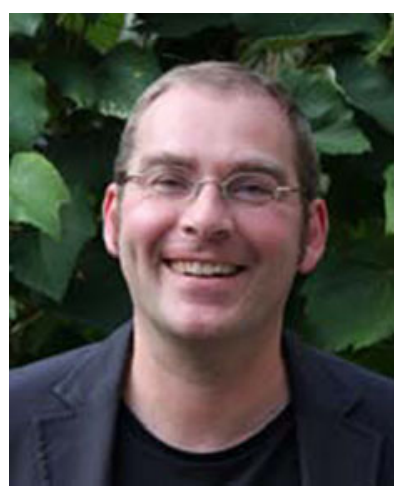

Wolfram Weckwerth

is Professor and Chair of the Department of Molecular Systems Biology at the University of Vienna (Austria). Prior to this, he was at the Max Planck Institute of Molecular Plant Physiology in Potsdam (Germany), where he established metabolomic methods and integrated proteomic and metabolic modelling approaches into a systems biology framework. He became the head of a research laboratory in the German FORSYS systems biology initiative before founding the Department of Molecular Systems Biology at the University of Vienna in 2008.

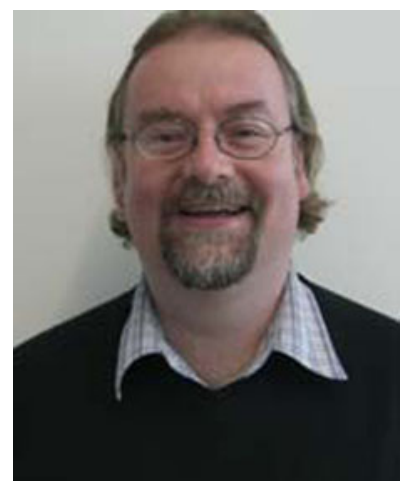

\section{Royston Goodacre}

is currently Professor of Biological Chemistry at the University of Manchester (UK). His group's main areas of research (http:// www.biospec.net/) are broadly within analytical biotechnology, metabolomics and systems biology. His expertise involves MS, Fourier transform IR spectroscopy and Raman spectroscopy, as well as advanced chemometrics, machine learning and evolutionary computational methods. He is Editor-in-Chief of the journal Metabolomics, a founding director of the Metabolomics Society and one of the directors of the Metabolic Profiling Forum. 